\title{
En Route to a Practical Primary Alcohol Deoxygenation
}

\author{
$\mathrm{Xi-Jie} \mathrm{Dai}{ }^{\dagger}$ and Chao-Jun $\mathrm{Li}^{*}{ }^{\dagger}$ \\ ${ }^{\dagger}$ Department of Chemistry and FQRNT Center for Green Chemistry and Catalysis, McGill University, 801 Sherbooke Street West, \\ Montreal, Quebec H3A 0B8, Canada
}

\section{Supporting Information}

ABSTRACT: A long-standing scientific challenge in the field of alcohol deoxygenation has been direct catalytic $\mathrm{sp}^{3} \mathrm{C}-\mathrm{O}$ defunctionalization with high selectivity and efficiency, in the presence of other functionalities, such as free hydroxyl groups and amines widely present in biological molecules. Previously, the selectivity issue had been only addressed by classic multistep deoxygenation strategies with stoichiometric reagents. Herein, we propose a catalytic late-transition-metal-catalyzed redox design, on the basis of dehydrogenation/Wolff-Kishner (WK) reduction, to simultaneously tackle the challenges regarding step economy and selectivity. The early development of our hypothesis focuses on an iridium-catalyzed process efficient mainly with activated alcohols, which dictates harsh reaction

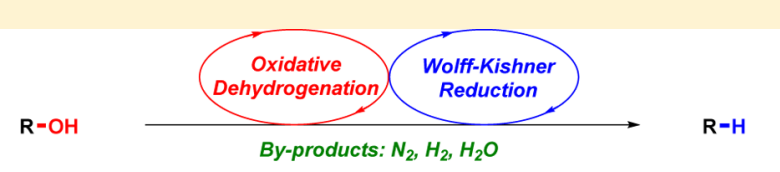

Highlighted Chemoselective Deoxygenation

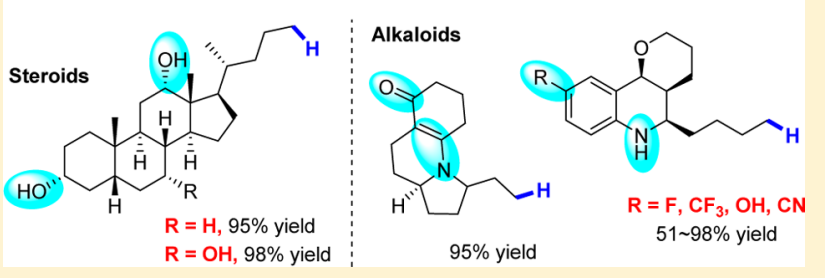
conditions and thus limits its synthetic utility. Later, a significant advancement has been made on aliphatic primary alcohol deoxygenation by employing a ruthenium complex, with good functional group tolerance and exclusive selectivity under practical reaction conditions. Its synthetic utility is further illustrated by excellent efficiency as well as complete chemo- and regioselectivity in both simple and complex molecular settings. Mechanistic discussion is also included with experimental supports. Overall, our current method successfully addresses the aforementioned challenges in the pertinent field, providing a practical redox-based approach to the direct $\mathrm{sp}^{3} \mathrm{C}-\mathrm{O}$ defunctionalization of aliphatic primary alcohols.

\section{INTRODUCTION}

Selective functionalization of a specific chemical bond in the molecule lies at the center of organic chemistry. Over the centuries, synthetic chemists have concentrated on the discovery of new reactions that allow facile construction of various chemical bonds in molecules to achieve complexity, which result in progressive advances in this field. ${ }^{1}$ In particular, late-stage interconversion of functional groups in high-value chemicals and drug candidates in a predictable and efficient manner enables rapid diversification and streamlines bioactive testing of closely related analogues. ${ }^{2}$ An equally crucial chemical transformation, yet historically being considered latent, is the opposite direction-selective defunctionalization. Its significance can be justified by fine-tuning of the desired properties of complex natural products and lead compounds (i.e., hydrogen-bond donors and acceptors, lipophilicity, etc.), especially at a late stage, when an accurate removal of a particular functional group is required without affecting others (Scheme 1A). Surprisingly, scarce endeavors have been geared toward this direction since the elaboration of functionalization methods at the beginning of the 21 st century.

A long-lasting challenge under such a context is how to selectively and directly remove $\mathrm{sp}^{3} \mathrm{C}-\mathrm{O}$ bonds from aliphatic alcohols, in the presence of other functionalities such as free hydroxyl groups and amines, without excessive chemical transformations. ${ }^{3}$
While the Barton-McCombie radical deoxygenation has been extensively utilized to remove sterically encumbered aliphatic hydroxyl groups in complex molecules (Scheme 1B, a) ${ }^{4}$ the deoxygenation of less sterically hindered alcohols is typically accomplished through the ionic reductive mechanism (Scheme 1B, b)..$^{5}$ The major downside of these classical methods is the requirement for multistep transformations, resulting in low step-efficiency. This raises an issue on inefficient chemical syntheses, particularly when it comes to the functional group interconversion in complex molecules at a late stage. ${ }^{6}$ To overcome this poor step economy, a few pioneering efforts in the 1990s have targeted direct deoxygenation of unhindered aliphatic alcohols, via $\mathrm{S}_{\mathrm{N}} 2$ or quasi- $S_{N} 2$ displacement using stoichiometric reagents (Scheme 1B, b). ${ }^{7}$ Nevertheless, limited functional group tolerance and poor selectivity generally render aforementioned strategies less synthetically attractive. To date, there is not a direct catalytic deoxygenation method for aliphatic alcohols with great selectivity and efficiency, especially one that is compatible with free hydroxyl groups and amines ubiquitously present in biological molecules, such as steroids and alkaloids.

In pursuit of such a deoxygenation protocol for aliphatic alcohols, we conceived a catalytic redox design based on dehydrogenation/Wolff-Kishner (WK) reduction using late-

Received: March 3, 2016

Published: April 5, 2016 
Scheme 1. (A) Synthetic Toolbox for Late-Stage Chemical Modification, (B) Classical Alcohol Deoxygenation and Unsolved Challenge, and C) Synopsis on Our Redox-Based, Catalytic, Direct Alcohol Deoxygenation

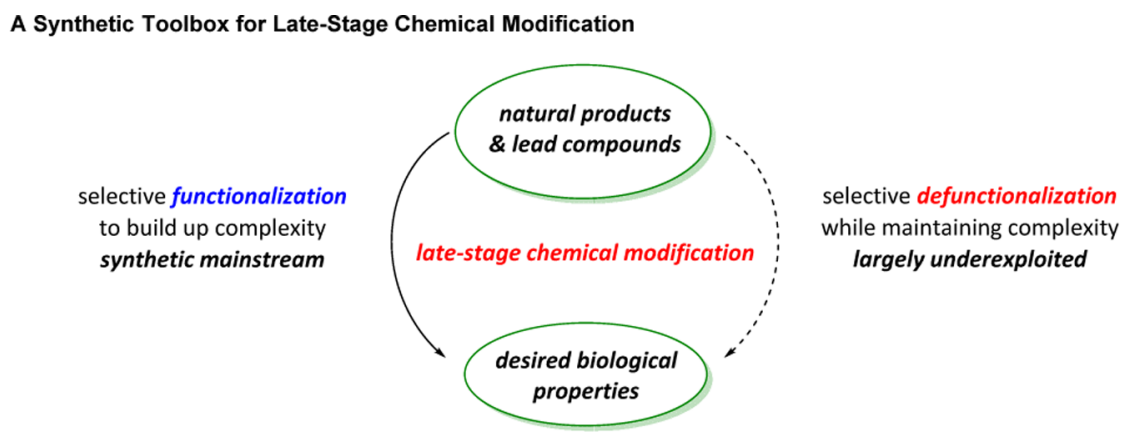

B Classical Alcohol Deoxygenation

a) Barton-McCombie Radical Pathway

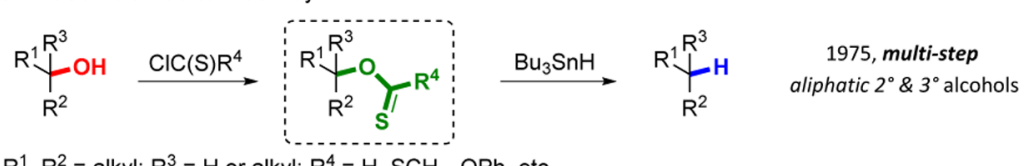

$R^{1}, R^{2}=$ alkyl; $R^{3}=H$ or alkyl; $R^{4}=H, S C H_{3}, O P h$, etc.

b) Ionic $S_{N} 2$-Based Pathway

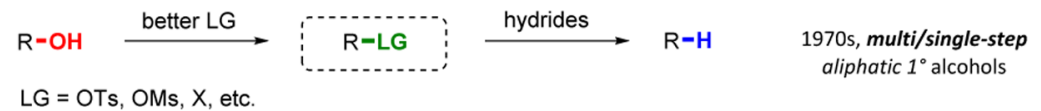

Unsolved Challenge: direct deoxygenation with high selectivity and efficiency?

C This Work: Redox-Based, Catalytic, Direct Alcohol Deoxygenation

a) Design Principle

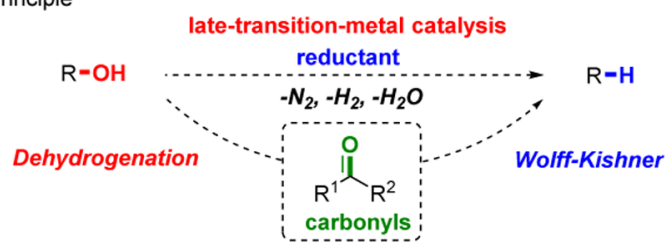

b) Key Features
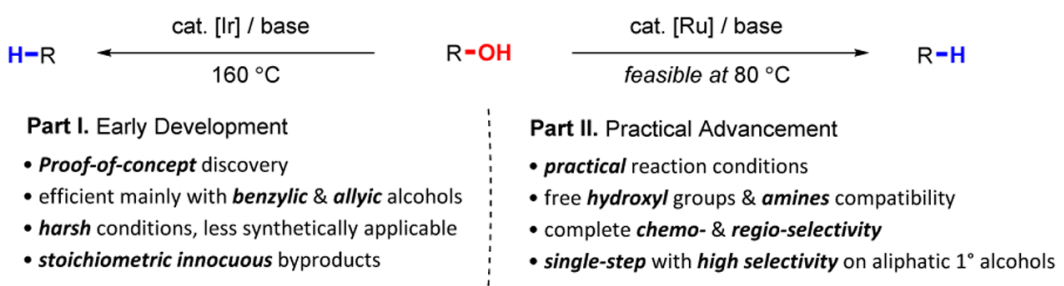

transition-metal catalysis (Scheme $1 \mathrm{C}$, a). We report herein a full development on this practical redox-based deoxygenation protocol. It contains two parts (Scheme 1C, b): (1) The first part focuses briefly on the early development on this method using an iridium catalyst and its major limitations; and (2) the second part discloses a significant practical advancement catalyzed by a ruthenium complex, with great efficiency and functional group selectivity, leading to a practical direct deoxygenation method for aliphatic primary alcohols. Its synthetic appeal lies in four key characteristics: (1) The reaction can be performed under practical conditions; (2) great functional group tolerance and excellent chemoselectivity in both simple and complex molecular settings, in particular leaving free hydroxyl groups and amines unaffected; (3) great regioselectivity, demonstrated by the monodeoxygenation of steroids with multiple cyclic secondary hydroxyl groups; and
(4) a synthetically benign strategy with stoichiometric nitrogen, hydrogen, and water as innocuous byproducts.

\section{REACTION DEVELOPMENT}

Following our catalytic redox design principle, we initially tested our hypothesis on piperonyl alcohol 1a using various iridium catalysts in the presence of base and hydrazine hydrate under thermal conditions. ${ }^{10}$ Gratifyingly, the corresponding deoxygenated product 3a was obtained in $32 \%$ yield after $12 \mathrm{~h}$ at $120{ }^{\circ} \mathrm{C}$ in water, when Vaska's complex $\left[\left(\mathrm{PPh}_{3}\right)_{2} \operatorname{IrCl}(\mathrm{CO})\right]$ and trimethylamine were employed (Table 1 , entry 1$)$. We then identified that methanol was a better protic solvent than water (Table 1, entry 2). Further optimization indicated that the stoichiometric quantity of strong base (i.e., $\mathrm{KOH}$ ) and excessive thermal energy were necessary for enhanced reactivity, which is in accordance with the classic WK reduction (Table 1, entry 3 ). A highly concentrated reaction solution was 
Table 1. Initial Optimization for Benzylic Alcohols ${ }^{a}$

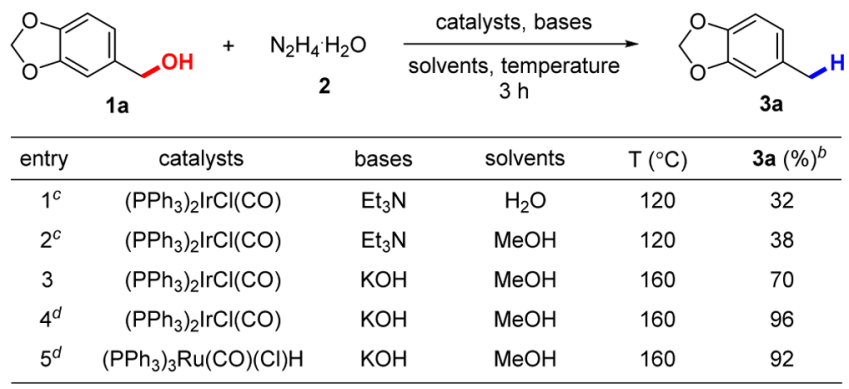

${ }^{a}$ Reaction conditions: 1a $(15 \mathrm{mg}, 0.1 \mathrm{mmol}), 2(10 \mathrm{mg}, 0.2 \mathrm{mmol})$, catalyst $(1 \mathrm{~mol} \%)$, bases $(0.2 \mathrm{mmol})$, sovlents $(0.1 \mathrm{~mL})$, under an air atmosphere. ${ }^{b} \mathrm{NMR}$ yields were determined using nitromethane as an internal standard. ${ }^{c}$ Reaction time: $12 \mathrm{~h} .{ }^{d} 10 \mu \mathrm{L} \mathrm{MeOH}$ was used as solvent.

also crucial to drive the reaction towards completion (Table 1, entry 4). We subsequently began testing a wide range of benzylic and allylic alcohols with this iridium-catalyzed deoxygenation protocol (Table 3, conditions A). In general, good to excellent yields were obtained for most activated substrates (3a-o, Table 3). Diminished reactivity, on the other hand, was observed for a few aliphatic substrates. ${ }^{10}$ At this stage, we thought that we had experimentally proved our catalytic redox-based hypothesis for a single-step deoxygenation process, providing a mechanistically distinct approach to address the issue of step economy. However, some critical aspects remained unsolved, preventing this preliminary iridiumbased strategy from being highly selective and synthetically viable. They were (1) significantly less reactivity shown in aliphatic alcohols (our ultimate target) than their benzylic and allylic counterparts, especially when other functional groups are present; (2) massive thermal input in a sealed reaction vessel $\left(\mathrm{MeOH}\right.$ at $160{ }^{\circ} \mathrm{C}$ with interior pressure built up over the course of the reaction); and (3) highly concentrated solution (10 M) which is not amenable to a practical scale-up, particularly with solid substrates. To circumvent these limitations, we reasoned that a different catalyst and a milder set of reaction conditions (less basic, lower temperature, pressure and concentration) could potentially lead to significant progress on the deoxygenation of aliphatic alcohols, resolving issues on both reactivity and chemoselectivity.

Practical Advancement. We thus embarked on the practical advancement of this catalytic redox-based deoxygenation method. Ruthenium-based complexes were selected as alternative catalysts because they were as effective as iridium complexes in our early studies (Table 1 , entry 4 vs 5), yet more cost-effective. Inspired by the modified low-temperature WK reduction, ${ }^{11}$ we investigated these conditions $(t-\mathrm{BuOH}$ as additive, DMSO as solvent), combining with the $[\mathrm{Ru}(p$ cymene $\left.\mathrm{Cl}_{2}\right]_{2} / \mathrm{dppb}\left(\mathbf{L}_{16}\right)$ catalytic system developed for the alcohol dehydrogenation. ${ }^{12}$ This combination allowed access to a trace amount of the desired product 5 a under relatively mild thermal conditions $\left(80{ }^{\circ} \mathrm{C}, 20 \mathrm{~h}\right)$ (Table 2 , entry 1$)$. This outcome was indeed encouraging, as such a low-temperature alcohol dehydrogenation had rarely been reported in the dehydrogenative oxidation studies, ${ }^{13}$ not to mention the more thermal demanding WK reduction. Against expectation, subsequent screenings of various catalysts, ligands, bases, or other parameters had little to no effect on reaction yields, suggesting a major impediment to the catalytic cycle. We
Table 2. Practical Optimization for Aliphatic Alcohols ${ }^{a}$

\begin{tabular}{|c|c|c|c|c|c|c|c|}
\hline & OH & & alysts, lig & nds, bases & & & \\
\hline & $4 a$ & 2 & olvents, T & $\left({ }^{\circ} \mathrm{C}\right), \mathrm{t}(\mathrm{h})$ & & & $5 a$ \\
\hline entry & catalyst & ligands & bases & solvents & $\mathrm{T}\left({ }^{\circ} \mathrm{C}\right)$ & $t(h)$ & $5 \mathbf{a}(\%)^{b}$ \\
\hline $1^{c}$ & {$\left[\mathrm{Ru}(p \text {-cymene }) \mathrm{Cl}_{2}\right]_{2}$} & $\operatorname{dppb}\left(L_{16}\right)$ & $t$-BuOK & DMSO & 80 & 20 & 8 \\
\hline 2 & {$\left[\mathrm{Ru}(p \text {-cymene }) \mathrm{Cl}_{2}\right]_{2}$} & $\operatorname{dppb}\left(L_{16}\right)$ & $t$-BuOK & $t$-BuOH & 80 & 9 & 33 \\
\hline 3 & $\left(\mathrm{PPh}_{3}\right)_{3} \operatorname{Ir}(\mathrm{CO}) \mathrm{Cl}$ & - & $t$-BuOK & $t-\mathrm{BuOH}$ & 80 & 9 & 5 \\
\hline 4 & $\mathrm{Ru}_{1}$ & - & $t$-BuOK & $t-\mathrm{BuOH}$ & 80 & 9 & 16 \\
\hline 5 & $\mathrm{Ru}_{2}$ & - & $t$-BuOK & $t$-BuOH & 80 & 9 & 13 \\
\hline 6 & $\mid \mathrm{r}_{1}$ & - & $t$-BuOK & $t-\mathrm{BuOH}$ & 80 & 9 & 9 \\
\hline 7 & $\mathrm{Ir}_{2}$ & - & $t$-BuOK & $t-\mathrm{BuOH}$ & 80 & 9 & 12 \\
\hline 8 & {$\left[\mathrm{Ru}(p \text {-cymene }) \mathrm{Cl}_{2}\right]_{2}$} & $\mathrm{I}^{\prime} \mathrm{PrS}\left(\mathrm{L}_{32}\right)$ & $t$-BuOK & $t-\mathrm{BuOH}$ & 80 & 9 & 10 \\
\hline 9 & {$\left[\mathrm{Ru}(p \text {-cymene }) \mathrm{Cl}_{2}\right]_{2}$} & TMEDA $\left(\mathrm{L}_{33}\right)$ & $t$-BuOK & $t-\mathrm{BuOH}$ & 80 & 9 & 7 \\
\hline 10 & {$\left[\mathrm{Ru}(p \text {-cymene }) \mathrm{Cl}_{2}\right]_{2}$} & $\operatorname{dppf}\left(\mathbf{L}_{24}\right)$ & $t$-BuOK & $t-\mathrm{BuOH}$ & 80 & 9 & 27 \\
\hline 11 & {$\left[\mathrm{Ru}(p \text {-cymene }) \mathrm{Cl}_{2}\right]_{2}$} & dmpe $\left(L_{14}\right)$ & $t$-BuOK & $t-\mathrm{BuOH}$ & 80 & 9 & 53 \\
\hline 12 & {$\left[\mathrm{Ru}(p \text {-cymene }) \mathrm{Cl}_{2}\right]_{2}$} & dmpe $\left(\mathbf{L}_{\mathbf{1 4}}\right)$ & $t$-BuOK & $t$-AmyOH & 80 & 9 & 37 \\
\hline $13^{d}$ & {$\left[\mathrm{Ru}(p \text {-cymene }) \mathrm{Cl}_{2}\right]_{2}$} & dmpe $\left(\mathbf{L}_{14}\right)$ & $\mathrm{K}_{3} \mathrm{PO}_{4}$ & $t-\mathrm{BuOH}$ & 100 & 4 & 19 \\
\hline $14^{d}$ & {$\left[\mathrm{Ru}(\mathrm{p} \text {-cymene }) \mathrm{Cl}_{2}\right]_{2}$} & dmpe $\left(L_{14}\right)$ & $t$-BuOK & $t-\mathrm{BuOH}$ & 100 & 4 & 67 \\
\hline $15^{d}$ & {$\left[\mathrm{Ru}(p \text {-cymene }) \mathrm{Cl}_{2}\right]_{2}$} & dmpe $\left(L_{14}\right)$ & $t$-BuOK & $t-\mathrm{BuOH}$ & 100 & 12 & 95 \\
\hline
\end{tabular}

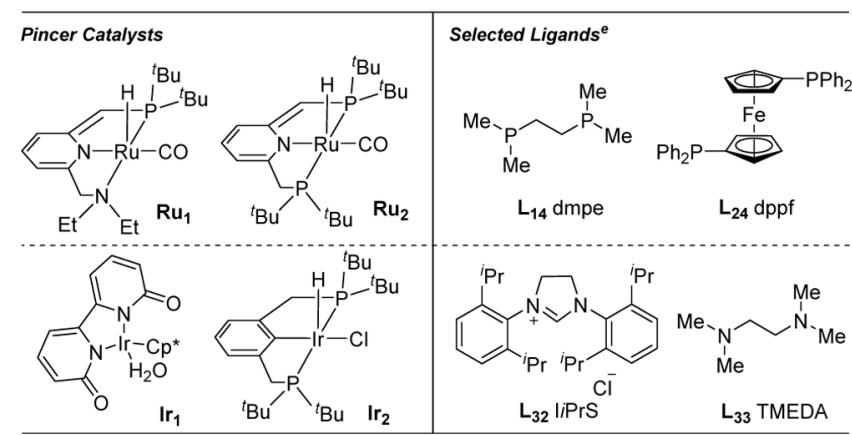

${ }^{a}$ General reaction conditions: $4 \mathrm{a}(0.2 \mathrm{mmol}),[\mathrm{Ru}]$ or $[\mathrm{Ir}]$ catalysts $(3$ mol \%), bidentate phosphine ligands (3 mol \%), 2 (0.24 mmol), bases $(0.1 \mathrm{mmol})$, DMSO $(0.28 \mathrm{mmol})$, solvents $(0.2 \mathrm{~mL})$, under Ar; see Tables S1-S12 in Supporting Information for details. ${ }^{b} \mathrm{NMR}$ yields were determined using 1,3,5-trimethoxybenzene as an internal standard. ${ }^{c} t-\mathrm{BuOH}(0.2 \mathrm{mmol}, 18 \mu \mathrm{L})$, DMSO $(0.4 \mathrm{~mL}) .{ }^{d} \mathrm{DMSO}$ $(20 \mathrm{~mol} \%, 2.8 \mu \mathrm{L}) .{ }^{e}$ See pages $\mathrm{S}-19$ and $\mathrm{S}-20$ in Supporting Information for all labelled ligands.

surmised that DMSO, a well-known dative ligand, ${ }^{14}$ might be problematic when it served as solvent. Consequently, saturation of the ruthenium complex by DMSO might leave the metal with no empty coordination site. To verify this assumption, the effects of various amounts of DMSO were examined (Table S5). Indeed, we were delighted to observe a large increase in yield as the volume of DMSO was decreased to $20 \mu \mathrm{L}(1.4$ equiv of $4 \mathbf{a}$ ) (Table 2, entry 2 ). Interestingly, the catalytic system consisting of $\left[\mathrm{Ru}(p \text {-cymene }) \mathrm{Cl}_{2}\right]_{2} /$ bidentate phosphine ligands outperformed the Vaska's complex (Table 2, entry 3), as well as other robust pincer-based ruthenium and iridium catalysts specifically designed for the acceptorless alcohol dehydrogenation (Table 2, entries 4-7; Table S1). ${ }^{15}$ A thorough ligand investigation followed (Table S3). Most monodentate phosphine ligands, regardless of their electronic or steric properties, performed poorly in the reaction. Neither tridentate phosphine ligands nor NHC ligands increased the yields (Table 2, entry 8 ). Some bidentate amido ligands, giving enhanced hydrogen production ${ }^{16}$ and showing great efficiency at low temperature, ${ }^{17}$ also failed under our conditions (Table 2, entry 9). The increased yields observed by the bidentate phosphine ligands (i.e., dppp $\left(\mathbf{L}_{15}\right)$, dppb $\left(\mathbf{L}_{16}\right)$, DIOP $\left(\mathbf{L}_{26}\right)$, etc.) with large bite angles led us to assume that bite angles 
Table 3. Substrate Scope of Redox-Based, Catalytic, Direct Deoxygenation

\begin{tabular}{|c|c|c|c|c|c|}
\hline \multirow{4}{*}{$3 a-0$} & $1 \mathrm{~mol} \%\left(\mathrm{PPh}_{3}\right)_{2} \mathrm{IrCl}(\mathrm{CO})$ & \multirow{2}{*}{$\mathrm{R} \curvearrowright \mathrm{OH}$} & \multirow{2}{*}{$\mathrm{N}_{2} \mathrm{H}_{4} \cdot \mathrm{H}_{2} \mathrm{O}$} & $\begin{array}{r}1.5 \mathrm{~mol} \%\left[\mathrm{Ru}(p \text {-cymene }) \mathrm{Cl}_{2}\right]_{2} \\
3 \mathrm{~mol} \% \mathrm{dmpe}, 20 \mathrm{~mol} \% \mathrm{DMSO}\end{array}$ & \\
\hline & $200 \mathrm{~mol} \% \mathrm{KOH}$ & & & $50 \mathrm{~mol} \% \mathrm{t}$-BuOK & \\
\hline & $\mathrm{MeOH}, 160^{\circ} \mathrm{C}, 3 \mathrm{~h}$ & 1a-o, $R=$ aryl or vinyl & 2 & $t-\mathrm{BuOH}, 100{ }^{\circ} \mathrm{C}, 12 \mathrm{~h}$ & $5 a-0$ \\
\hline & Condition A & 4a-o, R = alkyl & & Condition B & \\
\hline
\end{tabular}

Condition $\mathrm{A}^{\mathrm{a}}$ : benzylic \& allylic $1^{\circ}$ or $2^{\circ}$ alcohols

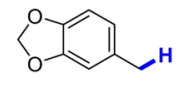

$\mathrm{MeO}$

$3 \mathbf{a}, 89 \%$<smiles>c1ccc(-c2ccccc2)cc1</smiles>

3f, $88 \%$<smiles>COc1ccc(C)cc1OC</smiles>

3k, $93 \%$

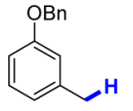

$3 c, 75 \%$

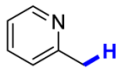

3h, $62 \%$

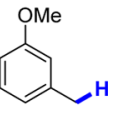

3m, $86 \%$

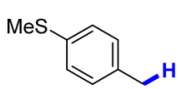

3d, $82 \%$

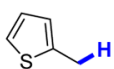

3i, $55 \%$

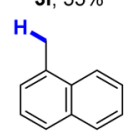

3n, $74 \%$

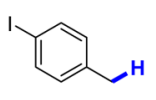

3e, $73 \%$

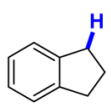

$3 \mathbf{j}, 43 \%$

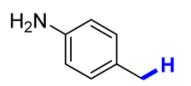

$30^{c}, 51 \%$

Condition $\mathrm{B}^{d}$ : aliphatic $1^{\circ}$ alcohols

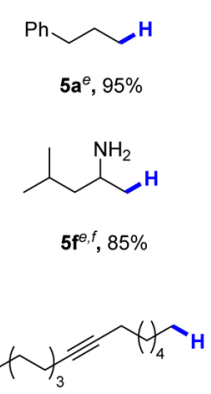

5k, $84 \%$

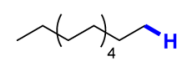

5b, $94 \%$<smiles>CC1CCCN1</smiles>

$\mathbf{5 g}^{e, f}, 83 \%$

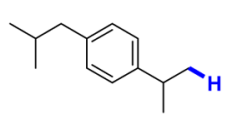

$\mathbf{5 1}^{g}, 79 \%$

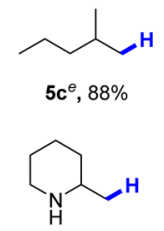

$5 h^{e}, 89 \%$

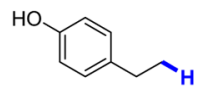

$5 \mathbf{m}^{g}, 65 \%$

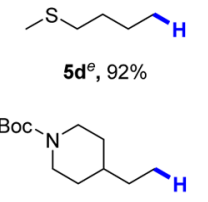

5i, $85 \%$

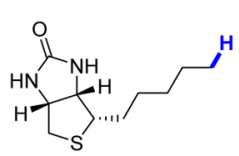

5n, $94 \%$

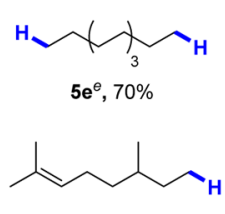

5j, $90 \%$

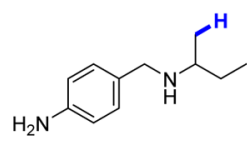

$50^{g, h}, 96 \%$

${ }^{a}$ Conditions A: 1a-o $(0.3 \mathrm{mmol}), 2(0.6 \mathrm{mmol}),\left(\mathrm{PPh}_{3}\right)_{2} \mathrm{lrCl}(\mathrm{CO})(2.3 \mathrm{mg}, 1 \mathrm{~mol} \%), \mathrm{KOH}(34 \mathrm{mg}, 0.6 \mathrm{mmol}), \mathrm{MeOH}(30 \mu \mathrm{L}), 160{ }^{\circ} \mathrm{C}, 3 \mathrm{~h}$, under an air atmosphere, isolated yields were obtained unless noted otherwise. ${ }^{b} \mathbf{1 g}$ : cinnamyl alcohol. ${ }^{c} 1$ o: 4 -nitrobenzyl alcohol. ${ }^{d}$ Conditions B: 4a-o $(0.2 \mathrm{mmol}),\left[\mathrm{Ru}(\mathrm{p} \text {-cymene }) \mathrm{Cl}_{2}\right]_{2}(1.5 \mathrm{~mol} \%)$, dmpe (3 mol \%), 2 (0.24 mmol), $t$-BuOK (0.1 mmol), DMSO $(20 \mathrm{~mol} \%), t$-BuOH $(0.2 \mathrm{~mL})$, $100{ }^{\circ} \mathrm{C}, 12 \mathrm{~h}$, under $\mathrm{Ar}$, isolated yields were obtained. ${ }^{e} \mathrm{NMR}$ yields were determined using 1,3,5-trimethoxybenzene as an internal standard. ${ }^{f}$ Racemization occured on both enantiomerically pure amino alcohols. ${ }^{g}$ Reaction time: 24 h. ${ }^{h}$ 4o: $N$-(4- $\{[(1-$ hydroxybutan-2-yl)amino $]$ methyl $\}$ phenyl)acetamide.

might be of importance to the catalyst's reactivity (Table 2, entry 10). ${ }^{18}$ Intriguingly, the use of 1,2-bis(dimethylphosphino)ethane (dmpe, $\mathbf{L}_{\mathbf{1 4}}$ ) provided the breakthrough in efficiency, affording 5a in 53\% NMR yield (Table 2, entry 11). The choice of protic polar solvent was required for the strong dipole-dipole interaction (hydrogen bonding) with DMSO. The best reactivity, however, was only accessible when $t$ - $\mathrm{BuOH}$ was used as solvent and DMSO as additive (Table 2, entry 11 vs 12; Tables S4 and S5), as opposed to their reverse roles described in the modified low-temperature WK studies. ${ }^{19}$ We also identified a concentration dependence as the dilute solution slowed down the reaction, leading to incomplete conversion within the same time frame (Table S6). To obtain an appreciable yield, the reaction could proceed at as low as 80 ${ }^{\circ} \mathrm{C}$, but further temperature decrease to $70{ }^{\circ} \mathrm{C}$ was associated with a significant attenuation of the yield (Table S7). While most inorganic bases were effective to promote the reaction, organic bases were largely ineffective (Table S8). The ultimate switch to $t$-BuOK resulted in the best yield (Table 2, entry 13 vs 14). Significantly, a substoichiometric amount of base was sufficient to promote this transformation, unlike the stoichiometric quantity required in the conventional WK reduction. An approximate linear kinetic relationship at $100{ }^{\circ} \mathrm{C}$ was established between the reaction yield and time, whereby $4 \mathrm{a}$ was mostly consumed within $4 \mathrm{~h}$ and completely in $12 \mathrm{~h}$ (Table 2 , entry 15; Table S10). It is of importance to note that neither dehydrating reagents nor anhydrous hydrazine sources can improve the reaction yield, given that the strictly anhydrous conditions are adopted in most WK-type reductions (Tables S11 and S12). ${ }^{11,19}$

\section{REACTION SCOPE}

Next, we applied this practical set of conditions to a wide range of aliphatic primary alcohols, furnishing good to excellent yields in nearly all cases (Table 3, conditions B). In particular, complete conversions were seen in all linear aliphatic primary alcohols, regardless of their backbone lengths (5a, 5b, 5e). Slightly lower yields were obtained for branched substrates with substituents at $\beta$ positions (5c, $\mathbf{5 1})$. In terms of functional group tolerance, thioether $(\mathbf{5 d})$, isolated internal double and triple bonds $(\mathbf{5 j}, \mathbf{5 k})$, phenolic hydroxyl group $(\mathbf{5 m})$, and Boc (tertbutyloxycarbonyl) (5i) all remained untouched. Amines are key moieties encountered in many pharmaceutical drugs and bioactive molecules. Their more nucleophilic nitrogen atoms 


\section{Scheme 2. Chemoselective Direct Deoxygenation in Complex Molecules}

A Sterically Encumbered Abietic Alcohol
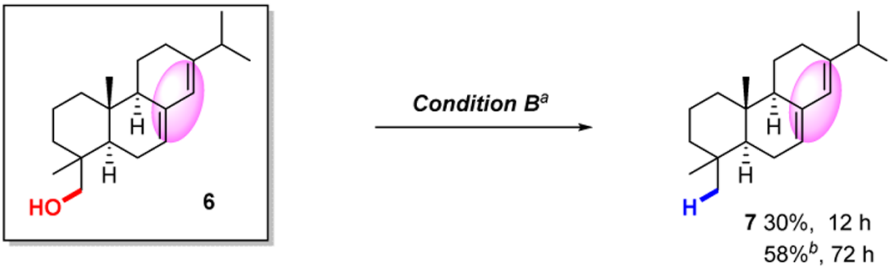

B Tricyclic Alkaloids with Conjugated Carbonyls
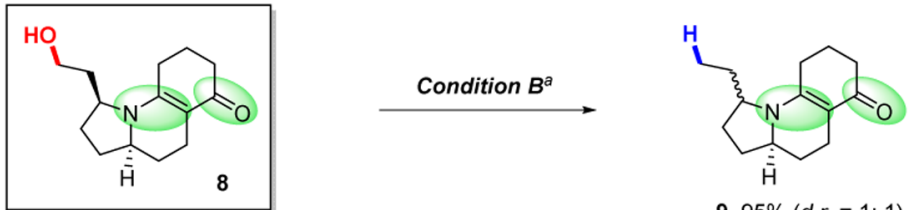

$9,95 \%($ d.r. $=1: 1)$

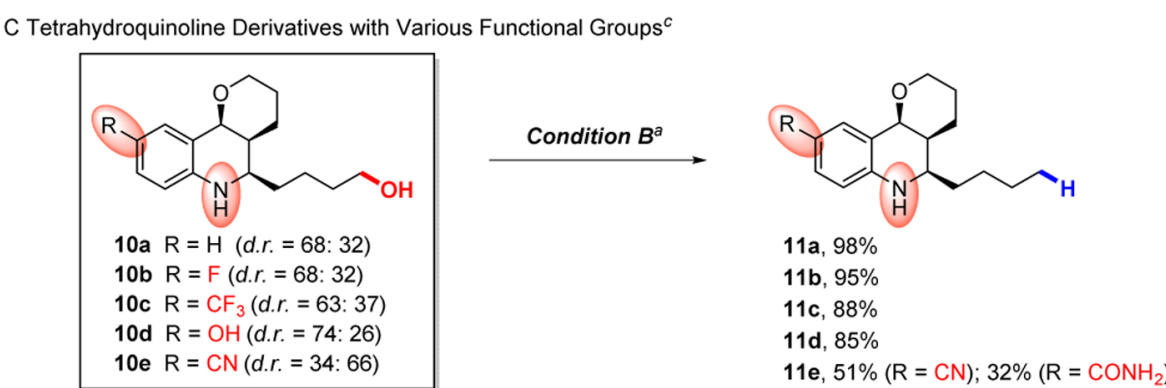

${ }^{a}$ Conditions B: 6, 8, and 10a-e $(0.1 \mathrm{mmol}),\left[\mathrm{Ru}(p \text {-cymene }) \mathrm{Cl}_{2}\right]_{2}(1.5 \mathrm{~mol} \%)$, dmpe (3 mol \%), 2 (0.24 mmol), $t$-BuOK (0.1 mmol), DMSO (20 $\mathrm{mol} \%), t-\mathrm{BuOH}(0.2 \mathrm{~mL}), 100{ }^{\circ} \mathrm{C}, 12 \mathrm{~h}$, under $\mathrm{Ar}$, isolated yields are obtained. ${ }^{b}\left[\mathrm{Ru}(p \text {-cymene }) \mathrm{Cl}_{2}\right]_{2}(3 \mathrm{~mol} \%), \mathrm{dmpe}(6 \mathrm{~mol} \%), 2(0.36 \mathrm{mmol})$. ${ }^{c}$ See ref 23 for the preparation of $10 a-e$.

usually make the chemoselective deoxygenation of aliphatic alcohols with amine functionalities (i.e., amino alcohols) problematic. This difficulty is even more profound when it comes to the direct deoxygenation protocol, and thereby no precedent exists under such circumstances. Gratifyingly, the direct cleavage of $\mathrm{C}-\mathrm{O}$ bonds occurred smoothly with great chemoselectivity in both acyclic amino alcohol $\mathbf{5 f}$ and cyclic amino alcohols $(\mathbf{5 g}, \mathbf{5 h})$, despite the fact that the $\mathrm{N}$-alkylation might have occured as a side reaction under ruthenium catalysis. $^{20}$ Excellent efficiency and chemoselectivity of our current practical protocol have also been demonstrated in bioactive molecules. For instance, $4 \mathbf{n}$ derived from (+)-biotin was quantitatively deoxygenated, without affecting the ureido and thiophene groups. However, functional groups labile to the basic conditions (i.e., amides, esters, etc.) tend to hydrolyze under current conditions. For instance, the deoxygenation of 4o was accompanied by the complete hydrolysis of the acyl group on its aniline moiety, giving rise to 50 in nearly quantitative yield. It is also noteworthy to mention that our catalytic redox design by and large suppressed the formation of azines ( $5 \%$ or less in most cases), which represents a major side reaction throughout all modified WK-type reductions. ${ }^{11,19}$

Chemoselective Direct Deoxygenation in Complex Molecules. Chemoselectivity is and has always been a major challenge in modern synthetic chemistry. ${ }^{21}$ To make our deoxygenation method more synthetically attractive, we decided to study the chemoseletivity in complex molecular settings. Abietic alcohol 6, possessing a conjugated double bond and a neopentyl quaternary carbon center, was chosen as our first target. Our initial attempt provided the deoxygenated product 7 in only $30 \%$ yield under the standard conditions. We reasoned that the sluggish reaction was potentially due to the sterically encumbered neopentyl quaternary carbon center in $\mathbf{6}$. As a result, it was difficult for the active ruthenium species to approach that carbon and perform an effective dehydrogenative oxidation. To restore reactivity, we doubled the amount of $\left[\mathrm{Ru}(p \text {-cymene }) \mathrm{Cl}_{2}\right]_{2}$ and dmpe $\left(\mathbf{L}_{14}\right)$, affording a reasonable $58 \%$ yield after a prolonged reaction time (Scheme $2 \mathrm{~A}$ ). Tricyclic heterocycle alkaloid $\mathbf{8}$, a key intermediate in the total synthesis of (+)-gephyrotoxin, ${ }^{22}$ has the $\alpha, \beta$-unsaturated carbonyl group in the molecule. The complete chemoselective deoxygenation was observed quantitatively in $\mathbf{8}$, albeit the epimerization occurred on its pyrrolidine ring (Scheme 2B). This epimerization is likely due to the sequential base-catalyzed retro-Michael/Michael addition processes. Rapid assembly of the tetrahydroquinoline skeleton and its structural modifications have attracted continuous synthetic interest over the past decades. Based on our early development of an $\mathrm{InCl}_{3}$-catalyzed protocol to synthesize tetrahydroquinoline alcohols, ${ }^{23}$ we now disclose their further structural modification using our deoxygenation method. All of these tetrahydroquinoline alcohols $(\mathbf{1 0 a}-\mathbf{e}$, Scheme 2C) displayed exceptional reactivity under current conditions, producing the corresponding deoxygenated tetrahydroquinoline derivatives in excellent yields, with the ratio of relative stereochemistry unchanged (11a-e, Scheme 2C). Equally important was the compatibility of varying functional groups on the aryl rings such as fluorine, trifluoromethane, and the phenolic hydroxyl group. Exception 
Scheme 3. Regioselective Direct Monodeoxygenation in Steroids

A Protecting Groups (PGs) Involved Chemistry
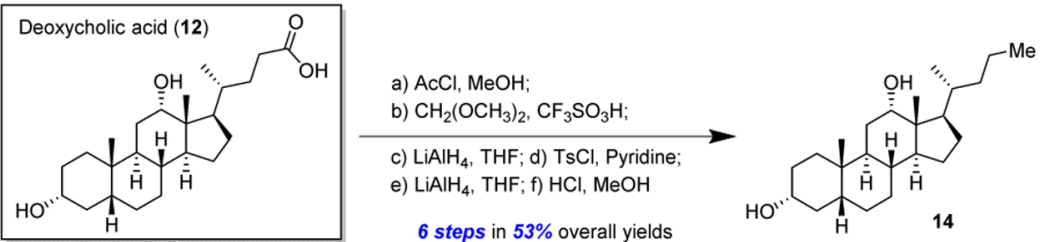

B PG-Free Approach with Our Deoxygenation Method

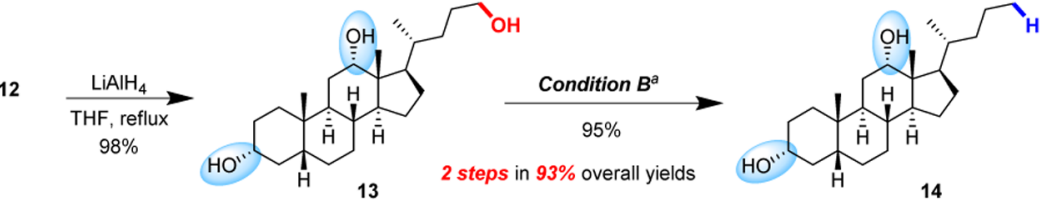

C Regioselective Deoxygenation of Cholic Alcohol

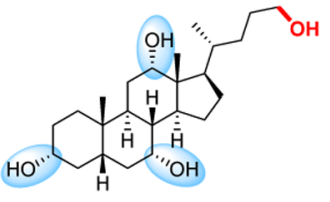

Cholic Alcohol (15)

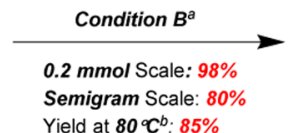

Yield at $80{ }^{\circ} C^{b}: 85 \%$

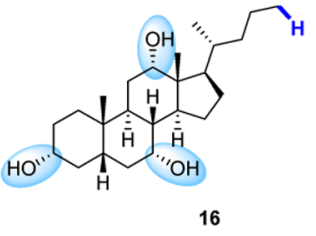

${ }^{a}$ Conditions B: 13 or $15(0.2 \mathrm{mmol}),\left[\mathrm{Ru}(p \text {-cymene }) \mathrm{Cl}_{2}\right]_{2}(1.5 \mathrm{~mol} \%)$, dmpe (3 mol \%), 2 (0.24 mmol), $t$-BuOK (0.1 mmol), DMSO (20 mol \%), $t$ - $\mathrm{BuOH}(0.4 \mathrm{~mL}), 100{ }^{\circ} \mathrm{C}, 12 \mathrm{~h}$, under Ar. ${ }^{b}$ Conditions: $15(0.1 \mathrm{mmol}),\left[\mathrm{Ru}(p \text {-cymene }) \mathrm{Cl}_{2}\right]_{2}(1.5 \mathrm{~mol} \%), \mathrm{dmpe}(3 \mathrm{~mol} \%), 2(0.12 \mathrm{mmol}), t-$ $\mathrm{BuOK}(0.05 \mathrm{mmol})$, DMSO $(20 \mathrm{~mol} \%), t-\mathrm{BuOH}(0.2 \mathrm{~mL}), 80{ }^{\circ} \mathrm{C}, 48 \mathrm{~h}$, under Ar.

\section{Scheme 4. Control Experiments To Yield Mechanistic Insights}

A Involvement of both $[\mathrm{Ru}]$ and dmpe

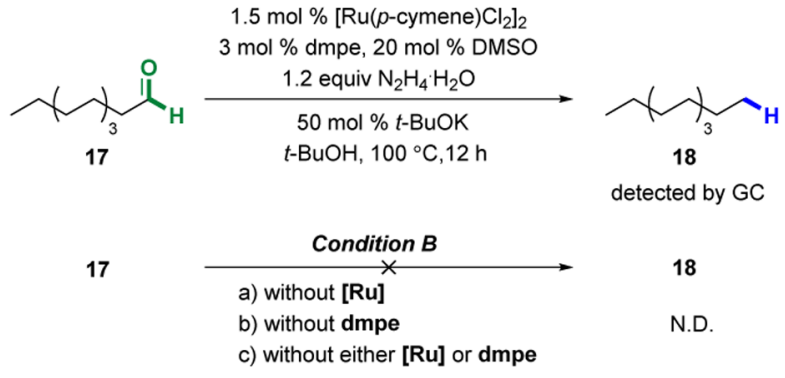

B Role of DMSO

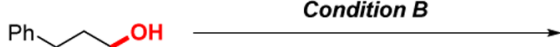

$4 a$

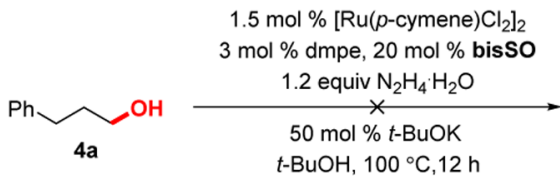<smiles>CCCCc1ccccc1</smiles>

$5 a$

$95 \%$ NMR yield

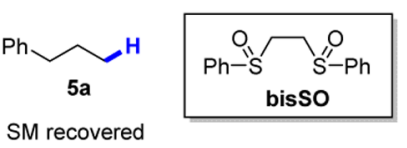

C Preformed Ru(dmpe $)_{2} \mathrm{Cl}_{2}$

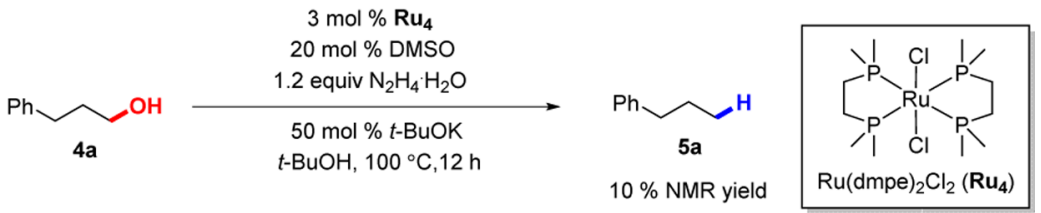


included the nitrile group in $\mathbf{1 0 e}$, as its partial hydrolysis to amide was observed. To summarize, this two-step synthetic approach allows a rapid and efficient access to deoxygenated tetrahydroquinoline derivatives for future biological studies, as some of their hydroxylated precursors have shown promising antitumor bioactivities. ${ }^{24}$

Regioselective Direct Monodeoxygenation in Steroids. Multiple hydroxyl groups in the same molecule raise an issue of regioselectivity when synthetic chemists try to selectively discriminate one hydroxyl group from another. On the basis of the relatively sluggish rate displayed by the sterically encumbered substrate 6, we envisaged the possibility of achieving regioselectivity through the pre-existing steric bias in natural products. Therefore, steroids became our targets of interest. Deoxycholic alcohol (13), which was easily obtained from deoxycholic acid (12) through a $\mathrm{LiAlH}_{4}$ reduction, contained three hydroxyl groups: one acyclic, primary; and two cyclic, secondary alcohols. Review of the literature indicated that no synthetic method was available to selectively deoxygenate $13 .^{25}$ Following our steric bias rationale, complete regioselectivity and remarkable reactivity were shown on the least sterically hindered acyclic primary hydroxyl group in $\mathbf{1 3}$. In comparison with a traditional synthetic route to access 14 (Scheme 3A), ${ }^{26}$ where protection-deprotection strategies are adapted in a six-step synthesis, our method features a two-step approach to obtain 14 in $93 \%$ yield (Scheme 3B). $3 \alpha, 7 \alpha, 12 \alpha$ Trihydroxycholane (16), another challenging steroidal derivative to synthesize, is an important precursor of seroflocculating agents and amphiphiles. ${ }^{27}$ Conventional synthetic approaches generate a mixture of over-reduced products. $^{28}$ Using our protocol, the monodeoxygenation of cholic alcohol (15) proceeded smoothly, furnishing $\mathbf{1 6}$ in quantitative yield on a small scale $(0.2 \mathrm{mmol})$ and slightly diminished yield on a semigram scale $(2 \mathrm{mmol})$. Remarkably, the monodeoxygenation of 15 took place smoothly at $80{ }^{\circ} \mathrm{C}$, giving 16 in $85 \%$ yield after $24 \mathrm{~h}$ (Scheme 3C).

\section{REACTION MECHANISM}

Several control experiments were conducted to shed light on the mechanism of this redox deoxygenation chemistry. Starting from decanal 17, decane 18 was captured by GC-MS under standard conditions, whereas no product was detected in the absence of $\left[\mathrm{Ru}(p \text {-cymene }) \mathrm{Cl}_{2}\right]_{2}$ and dmpe. This result suggested the critical involvement of both metal and ligand in the reductive transformation (Scheme $4 \mathrm{~A}$ ). To better understand the exact role of DMSO, a structurally similar bisSO ligand (1,2-bis(phenylsulfinyl)ethane) was tested, yet no product was observed (Scheme 4B). Accordingly, it appeared that DMSO was more than just a ligand in our current catalytic system. Interestingly, the use of presynthesized $\mathrm{Ru}(\mathrm{dmpe})_{2} \mathrm{Cl}_{2}$ $\left(\mathrm{Ru}_{4}\right)$ dramatically decreased the overall efficiency, indicating that it was not the active metal species (Scheme 4C). We also confirmed the production of hydrogen and nitrogen gas over the course of the reaction, by analyzing the headspace of the reaction vial with GC-MS.

We tentatively postulate a mechanism based on all our experimental data, in addition to studies from others (Scheme 5). ${ }^{10-12,15 a}$ According to the widely accepted mechanism associated with the alcohol amination catalyzed by a $[\mathrm{Ru}(p$ cymene $\left.\mathrm{Cl}_{2}\right]_{2}$ /diphosphine system, the bidentate phosphine coordinated complex $\mathbf{A}$ is initially generated by a ligand exchange of $\left[\mathrm{Ru}(p \text {-cymene }) \mathrm{Cl}_{2}\right]_{2}$ with $\mathrm{L}_{\mathbf{1 4}}$. A ruthenium $(0)$ complex $\mathbf{B}$ can then be formed via an alcohol association
Scheme 5. Proposed Catalytic Cycle

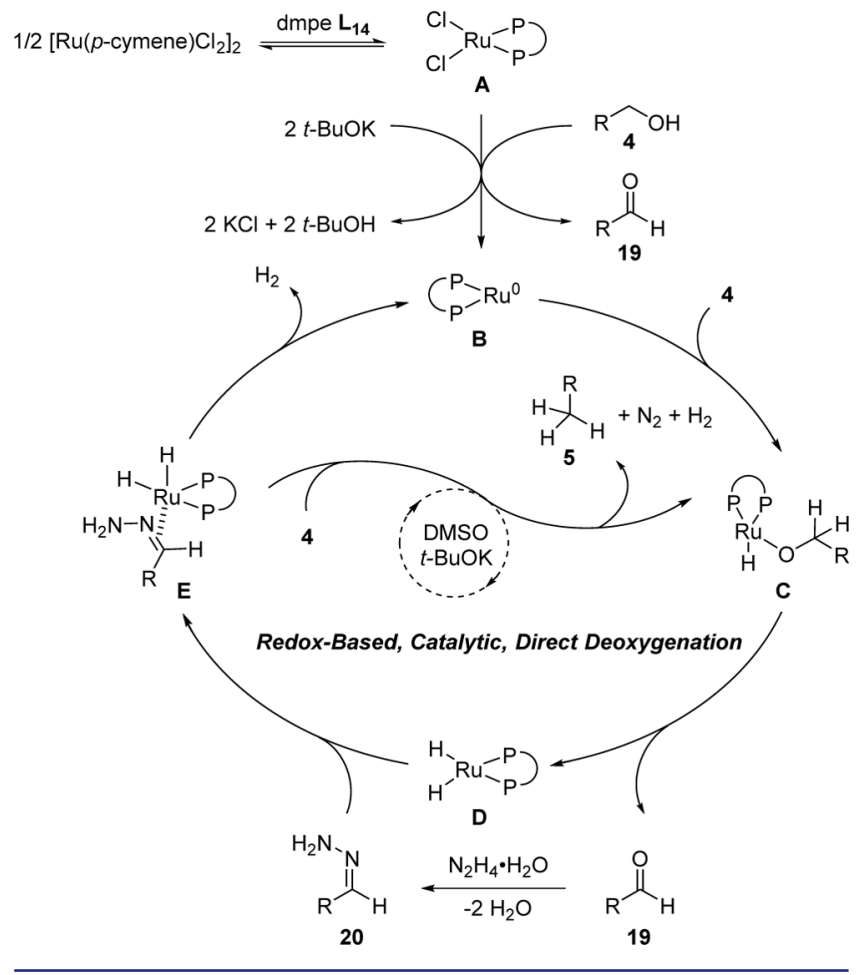

followed by the loss of $\mathrm{HCl}$ in the presence of base. Oxidative addition of the alcohol provides the alkoxy hydride complex $\mathbf{C}$, which further undergoes $\beta$-hydride elimination to produce the chelated aldehyde complex. Dissociation of aldehyde 19, hydrazone formation, and association of hydrazone 20 to ruthenium dihydride complex $\mathbf{D}$ provide $\mathbf{E}$. Its reductive decomposition, in the presence of DMSO and $t$-BuOK, leads to the desired deoxygenated product $\mathbf{5 a}$, with concomitant release of hydrogen and nitrogen gas. The regeneration of $\mathbf{B}$ presumably goes through the reductive elimination of $\mathbf{E}$. Unlike all low-temperature modified WK reduction, only a catalytic amount of DMSO and substoichiometric amount of $t$ $\mathrm{BuOK}$ are required in our method, suggesting an unconventional rate acceleration due to the participation of $[\mathrm{Ru}(p$ cymene) $\left.\mathrm{Cl}_{2}\right]_{2}$ and dmpe.

\section{CONCLUSIONS}

In conclusion, we have discovered and developed a direct catalytic alcohol deoxygenation protocol based on redox chemistry. Our early studies show that deoxygenation of benzylic and allylic alcohols can be realized using iridium catalysis in single-step via a sequential redox process (dehydrogenation/WK reduction). However, the harsh reaction conditions and limited scope make it less synthetically applicable. Our later development catalyzed by the ruthenium catalysis focuses on aliphatic primary alcohols, solving the selectivity issue (both chemo- and regio-selectivity) under practical conditions. We have demonstrated its superb synthetic viability, both efficiency and selectivity, using molecules with varying degrees of complexity and a number of different functional groups. Significantly, our current method can be successfully implemented on the highly chemoselective direct deoxygenation of alkaloids and regioselective direct monodeoxygenation of steroids with multiple secondary hydroxyl groups on both small and semigram scales. Striking features of 
our method also include relatively mild thermal conditions as well as innocuous byproducts. The indispensable roles of ruthenium catalyst, bidentate phosphine ligand dmpe $\left(\mathrm{L}_{14}\right)$, a catalytic amount of DMSO, and a substoichiometric amount of base imply a transition-metal-assisted reductive WK transformation. While further experimental evidence is required to elucidate the mechanism of this deoxygenation chemistry, our new redox-based approach has great potential to become a useful synthetic tool for the direct $\mathrm{sp}^{3} \mathrm{C}-\mathrm{O}$ defunctionalization in complex molecules.

\section{ASSOCIATED CONTENT}

\section{S Supporting Information}

The Supporting Information is available free of charge on the ACS Publications website at DOI: 10.1021/jacs.6b02344.

Experimental details and spectroscopic data for all products (PDF)

\section{AUTHOR INFORMATION}

\section{Corresponding Author}

*cj.li@mcgill.ca

\section{Notes}

The authors declare no competing financial interest.

\section{ACKNOWLEDGMENTS}

The authors acknowledge the Canada Research Chair Foundation (to C.-J.L.), the CFI, FQRNT Center for Green Chemistry and Catalysis, NSERC, and McGill University for financial support. Dr. Zheng-Wang Chen and Dr. Feng Wang are greatly appreciated for their generous helps on preparation of some alcohol substrates. We acknowledge Dr. Jian-Lin Huang on his initial discovery of the deoxygenated product using the iridium catalysis. We acknowledge a generous sample donation of compound $\mathbf{8}$ from Professor Claude Spino at Université de Sherbrooke. X.-J.D. thanks the chemistry department for a doctoral fellowship (Molson \& Hilton Hart fellowship).

\section{REFERENCES}

(1) (a) Labinger, J. A.; Bercaw, J. E. Nature 2002, 417, 507-514. (b) Godula, K.; Sames, D. Science 2006, 312, 67-72.

(2) (a) Wencel-Delord, J.; Glorius, F. Nat. Chem. 2013, 5, 369-375.

(b) Dai, H.-X.; Stepan, A. F.; Plummer, M. S.; Zhang, Y.-H.; Yu, J.-Q. J. Am. Chem. Soc. 2011, 133, 7222-7228.

(3) Wender, P. A.; Verma, V. A.; Paxton, T. J.; Pillow, T. H. Acc. Chem. Res. 2008, 41, 40-49.

(4) (a) Barton, D. H. R; McCombie, S. W. J. Chem. Soc., Perkin Trans. 1 1975, 1574-1585. (b) Hartwig, W. Tetrahedron 1983, 39, 2609-2645. (c) Robins, M. J.; Wilson, J. S.; Hansske, F. J. Am. Chem. Soc. 1983, 105, 4059-4065.

(5) (a) Masamune, S.; Rossy, P. A.; Bates, G. S. J. Am. Chem. Soc. 1973, 95, 6452-6454. (b) Masamune, S.; Bates, G. S.; Georghiou, P. E. J. Am. Chem. Soc. 1974, 96, 3686-3688.

(6) Our current method (condition B), with great chemoselectivity in steroids, can potentially increase the overall step-efficiency in the total synthesis of humulene, starting from compound $\mathbf{1 0}$ in the paper: Kitagawa, Y.; Itoh, A.; Hashimoto, S.; Yamamoto, H.; Nozaki, H. J. J. Am. Chem. Soc. 1977, 99, 3864-3867.

(7) (a) Hendrickson, J. B.; Singer, M.; Hussoin, M. S. J. Org. Chem. 1993, 58, 6913-6914. (b) Gevorgyan, V.; Rubin, M.; Benson, S.; Liu, J.-X.; Yamamoto, Y. J. Org. Chem. 2000, 65, 6179-6186. (c) Gevorgyan, V.; Liu, J.-X.; Rubin, M.; Benson, S.; Yamamoto, Y. Tetrahedron Lett. 1999, 40, 8919-8922. (d) Myers, A. G.; Movassaghi, M.; Zheng, B. J. Am. Chem. Soc. 1997, 119, 8572-8573.
(8) Herrmann, J. M.; König, B. Eur. J. Org. Chem. 2013, 7017-7027. (b) Trost, B. M. Science 1983, 219, 245-250.

(9) Anastas, P. T.; Warner, J. C. In Green Chemistry: Theory and Practice; Oxford University Press: Oxford, 2000.

(10) For details on our early development of iridium-catalyzed direct deoxygenation method, see: Huang, J.-L.; Dai, X.-J.; Li, C.-J. Eur. J. Org. Chem. 2013, 6496-6500.

(11) (a) Cram, D. J.; Sahyun, M. R. V.; Knox, G. R. J. Am. Chem. Soc. 1962, 84, 1734-1735. (b) Grundon, M. F.; Henbest, H. B.; Scott, M. D. J. Chem. Soc. 1963, 1855-1858. (c) Furrow, M. E.; Myers, A. G. J. Am. Chem. Soc. 2004, 126, 5436-5445.

(12) For reviews in alcohol dehydrogenation, see: (a) Ledger, A. E. W.; Slatford, P. A.; Lowe, J. P.; Mahon, M. F.; Whittlesey, M. K.; Williams, J. M. J. Dalton Trans. 2009, 716-722. (b) Bower, J. F.; Kim, I. S.; Patman, R. L.; Krische, M. J. Angew. Chem., Int. Ed. 2009, 48, 3446. (c) Hamid, M. H. S. A.; Slatford, P. A.; Williams, J. M. J. Adv. Synth. Catal. 2007, 349, 1555-1575. (d) Dobereiner, G. E.; Crabtree, R. H. Chem. Rev. 2010, 110, 681-703.

(13) (a) Zhang, J.; Gandelman, M.; Shimon, L. J. W.; Rozenberg, H.; Milstein, D. Organometallics 2004, 23, 4026-4033. (b) Kawahara, R.; Fujita, K.-i.; Yamaguchi, R. Angew. Chem., Int. Ed. 2012, 51, 1279012794. (c) Baratta, W.; Bossi, G.; Putignano, E.; Rigo, P. Chem. - Eur. J. 2011, 17, 3474-3481.

(14) Calligaris, M. Coord. Chem. Rev. 2004, 248, 351-375.

(15) (a) Hamid, M. H. S. A.; Allen, C. L.; Lamb, G. W.; Maxwell, A. C.; Maytum, H. C.; Watson, A. J. A.; Williams, J. M. J. J. Am. Chem. Soc. 2009, 131, 1766-1774. (b) Gunanathan, C.; Milstein, D. Science 2013, 341, 1229712. (c) Gunanathan, C.; Ben-David, Y.; Milstein, D. Science 2007, 317, 790-792. (d) Kawahara, R.; Fujita, K.-i.; Yamaguchi, R. J. Am. Chem. Soc. 2012, 134, 3643-3646.

(16) Junge, H.; Loges, B.; Beller, M. Chem. Commun. 2007, 522-524.

(17) Enyong, A. B.; Moasser, B. J. Org. Chem. 2014, 79, 7553-7563.

(18) Birkholz, M.-N.; Freixa, Z.; van Leeuwen, P. W. N. M. Chem. Soc. Rev. 2009, 38, 1099-1118.

(19) (a) Szmant, H. H.; Román, M. N. J. Am. Chem. Soc. 1966, 88, 4034-4039. (b) Wallace, T. J.; Hofmann, J. E.; Schriesheim, A. J. Am. Chem. Soc. 1963, 85, 2739-2743.

(20) (a) Nixon, T. D.; Whittlesey, M. K.; Williams, J. M. J. Dalton Trans. 2009, 753-762. (b) Guillena, G.; Ramón, D. J.; Yus, M. Angew. Chem., Int. Ed. 2007, 46, 2358-2364.

(21) Afagh, N. A.; Yudin, A. K. Angew. Chem., Int. Ed. 2010, 49, 262310.

(22) Wei, L.-L.; Hsung, R. P.; Sklenicka, H. M.; Gerasyuto, A. I. Angew. Chem., Int. Ed. 2001, 40, 1516-1518.

(23) Zhang, J.; Li, C.-J. J. Org. Chem. 2002, 67, 3969-3971.

(24) Li, C.-J.; Zhang, J. Methods for synthesizing heterocycles and therapeutic use of the heterocycles for cancers. U.S. Patent 7250423, July 31, 2007.

(25) While methods for the selective deoxygenation of $\mathbf{1 3}$ are not available, monodeoxygenation of a similar steroid compound has been reported, see: Denancé, M.; Guyot, M.; Samadi, M. Steroids 2006, 71, $599-602$.

(26) On, J. H.; Cho, K. T.; Park, Y.; Hahm, S.; Kim, W.; Cho, J. Y.; Hwang, J. H.; Jun, Y. M.; Cha, G. S.; Nam, H.; Kim, B. H. Tetrahedron 2009, 65, 1415-1423.

(27) (a) Blickenstaff, R. T.; Chang, F. C. J. Am. Chem. Soc. 1959, 81, 2835-2838. (b) Lee, S. C.; Bennett, B. C.; Hong, W.-X.; Fu, Y.; Baker, K. A.; Marcoux, J.; Robinson, C. V.; Ward, A. B.; Halpert, J. R.; Stevens, R. C.; Stout, C. D.; Yeager, M. J.; Zhang, Q. Proc. Natl. Acad. Sci. U. S. A. 2013, 110, E1203-E1211.

(28) Zhang, Q.; Ma, X.; Ward, A.; Hong, W.-X.; Jaakola, V.-P.; Stevens, R. C.; Finn, M. G.; Chang, G. Angew. Chem., Int. Ed. 2007, 46, $7023-7025$ 